\title{
Percepción por los docentes de la Facultad de Medicina y Odontología de la USC sobre la docencia virtual y sincrónica tras la crisis del Covid-19
}

\section{Perception by teachers of the Faculty of Medicine and Dentistry of the USC on virtual and synchronous teaching after the COVID-19 crisis}

\section{Mario Pérez-Sayáns ${ }^{1,2}$, Cintia M. Chamorro' ${ }^{2}$, Dolores Reboiras ${ }^{1}$, Pilar Gándara ${ }^{1,2}$ y Mercedes Gallas ${ }^{1}$.}

1 Departamento de Cirugía y especialidades médico quirúrgicas. Área Estomatología. Facultad de Medicina y Odontología; mario.perez@usc.es ORCID ID: 0000-0003-21969868; mariadolores.reboiras@usc.es ORCID ID: 0000-0001-5792-8350; pilar.gandara@usc.es ORCID ID: 0000-0003-2259-7971; mercedes.gallas.torreira@usc.es ORCID ID: 0000-0003-4625-3321

2 Instituto de Investigación Sanitaria de Santiago de Compostela (IDID); cintiamica.chamorro@rai.usc.es, ORCID ID: 0000-0002-5637-9908

* Correspondencia: perezsayans@gmail.com

Recibido: 1-10-2020; Aceptado: 19-10-2020; Publicado: 20-10-2020

Resumen: La pandemia del Covid-19 ha impulsado cambios en la educación superior mundialmente, exigidos por las medidas de distanciamiento social que impiden cualquier enseñanza presencial. El objetivo de este trabajo es conocer la percepción y uso por los docentes de la Facultad de Medicina y Odontología de la Universidad de Santiago de Compostela (USC) sobre el entorno de aprendizaje virtual (VLE) mediante un estudio transversal basado en encuestas autoaplicadas con Microsoft Forms. Los datos se analizaron estadísticamente mediante el programa SPSS v.24.0. La tasa de respuesta fue del $46.3 \%$. La muestra estaba formada por 23 hombres (35.5\%) y 40 mujeres (63.5 \%). El 50.8 \% tiene más de 20 años de experiencia docente. El $49.2 \%$ de los encuestados no utilizaba el campus virtual (CV) antes de la crisis del Covid-19 y el 6.3 \% continúa sin usarlo actualmente. Sólo un $6.3 \%$ utilizaba la docencia en remoto síncrona y sólo el 54 \% cree es válida para su aplicación a las Ciencias de la Salud. Los no usuarios del CV, mayoritariamente $(64.5 \%)$ comienzan a usarlo como mensajería y entrega de material docente, mientras que de los usuarios asiduos un $59.4 \%$ explotan todas sus herramientas $\left(\mathrm{chi}^{2}=15.640, \mathrm{~V}\right.$ de Cramer $=0.498, \mathrm{p}=0.001)$. Los registros de uso del estudio constatan una baja utilización en el entorno docente analizado indicando que la adquisición de capacitación en el uso del campus virtual en la docencia universitaria debería ser promocionada al objeto de incrementar su empleo.

Palabras clave: entorno de aprendizaje virtual, educación en ciencias de la salud, estilos de aprendizaje, enseñanza, covid-19.

\begin{abstract}
The Covid-19 pandemic has promoted changes in higher education worldwide, demanded by social distancing measures that prevent any face-to-face teaching. The objective of this work is to analyze the perception and use by professors of the Faculty of Medicine and Dentistry of the University of Santiago de
\end{abstract}


Compostela (USC) on the virtual learning environment (VLE) through a crosssectional study based on self-administered surveys using Microsoft Forms. The data were statistically analyzed using the SPSS v.24.0 program. The response rate was $46.3 \%$. The sample consisted of 23 men (35.5\%) and 40 women (63.5\%). 50.8\% have more than 20 years of teaching experience. $49.2 \%$ of those surveyed did not use the virtual campus (CV) before the Covid-19 crisis and 6.3\% continue not to use it today. Only $6.3 \%$ used synchronous remote teaching and only $54 \%$ believe it is valid for its application to Health Sciences. Most of the non-users of the CV (64.5\%) begin to use it as courier and delivery of teaching material, while regarding regular users, $59.4 \%$ exploit all its tools (chi2 $=15.640$, Cramer's $\mathrm{V}=0.498, \mathrm{p}=0.001$ ). The records of use of the study confirm a low use in the analyzed teaching environment, indicating that the acquisition of training in the use of the virtual campus in university teaching should be promoted in order to increase their utilization.

Keywords: virtual learning environment, health sciences education, learning styles, teaching, covid-19.

\section{Introducción}

La crisis sanitaria COVID-19 supuso la interrupción de la docencia presencial en el segundo cuatrimestre del curso 2019-20 sobre todo en las instituciones educativas que no contaban con un modelo no presencial y/o semi-presencial instaurado de formación a distancia (1).

La formación en ciencias de la salud está cambiando debido a la era digital (2). Los sistemas de gestión del aprendizaje (siglas LMS) están ampliando la accesibilidad a la capacitación a profesionales y estudiantes (3). El entorno de aprendizaje virtual (VLE en inglés) ofrece muchas ventajas al estudiantado: horarios flexibles, eliminación del tiempo de viaje y fomento de la interacción alumnado-educador (4). La educación en línea, tanto síncrona como asíncrona, conecta estudiantes y profesores geográficamente, haciéndola más universal y accesible, sustituyendo, parcialmente, el aprendizaje presencial (5). Algunas desventajas de los cursos en línea para el estudiante son la sensación de aislamiento y la dificultad de adaptación (6). Para los docentes, los contenidos no varían de un tipo a otro de formación, sin embargo la forma en línea agrega un matiz de complejidad (7).

Los LMS engloban una serie diversa de canales de comunicación asíncronos y sincrónicos (8). Existen muchas plataformas a distancia verificadas para la formación en ciencias de la salud, las más populares son Blackboard Academic Suite $(5,9)$ y Moodle $(10,11)$. Todas usan herramientas interactivas formativas, así como sumativas que fomentan el aprendizaje colaborativo y el pensamiento crítico (12-15). Aunque disponibles para el profesorado, su uso y aprovechamiento durante la crisis del Covid-19 se ha visto modificado sustancialmente. Por ello nuestro objetivo es conocer la percepción y el uso por parte de los docentes de la Facultad de Medicina y Odontología de la USC de la docencia virtual asíncrona y en remoto durante el confinamiento en la crisis del Covid-19 en el curso académico 2019-20.

\section{Métodos}

Este estudio transversal se realizó siguiendo las recomendaciones de la guía STROBE (16). El comité autonómico de ética en la investigación ha 
eximido del pertinente informe en su tramitación pues este cumple los requisitos éticos exigidos al no recoger datos de salud (Ref. 2020/192). Todos los profesores de la Facultad de Medicina y Odontología de la Universidad de Santiago de Compostela (USC) fueron invitados a participar. Para calcular la potencia del estudio utilizamos Epidat 4.2 (SERGAS, Galicia, España). Con muestra total de 136 profesores, asumiendo una heterogeneidad del $50 \%$ con una tasa de respuesta de 63 profesores (46.3\%), la potencia estadística es del $72 \%$ con un margen de error del $28 \%$.

Diseñamos una encuesta autoaplicada (Microsoft Forms Office 365; Microsoft, Madrid, España) formada por preguntas cerradas, respuesta única y/o múltiple, preguntas modalidad escala Likert y formato libre, que difundida por correo electrónico se estructura en: (I) Datos sociodemográficos (edad, género), (II) Perfil Docente (Área de Conocimiento, Experiencia docente, Categoría profesional, Cursos, Grado/Postgrado, número de materias y créditos totales), (III) Manejo Campus Virtual (CV) (Uso previo, tiempo de dedicación, frecuencia de acceso, forma de explotación, participación estudiantil), (IV) Docencia en remoto no presencial (uso previo sistemas de videollamada, implicación del alumnado, participación activa, problemas de conexión, otros métodos), (V) Evaluación personal (grado de satisfacción, adecuación a ciencias de la salud y uso como método único).

Una encuesta piloto fue probada en una muestra al azar de 10 profesores para evaluar fiabilidad, exhaustividad y tiempo de implementación (tiempo medio $7 \mathrm{~min}$ ). Para conocer la fiabilidad final empleamos la prueba alfa de Cronbach. Se utilizó la estratificación en bloques temáticos (Tabla 1) y el valor medio para este cuestionario fue de 0,84 (fiabilidad moderada).

Los datos recogidos en una base de datos diseñada específicamente se analizaron estadísticamente mediante SPSS v.24.0 (IBM, Statistics, NY, USA) estableciéndose tablas de contingencia mediante el test chi cuadrado y el grado de dependencia mediante la $\mathrm{V}$ de Cramer. Para la comparativa número de materias impartidas en relación al uso CV se utilizó el test ANOVA para comparación de medias con un nivel de significación $\mathrm{p} \leq 0.05$.

\section{Resultados}

La muestra de docentes participantes estaba compuesta por 23 hombres (35.5\%) y 40 mujeres (63.5\%) mayoritariamente con edades entre 56-70 años (42.9\%) (Tabla 1).

Tabla 1. Resumen de datos descriptivos del estudio.

\begin{tabular}{|c|c|}
\hline Variable & $\mathrm{N}(\%)$ \\
\hline Género $(\mathrm{N}$ total=63) & \\
\hline Hombre & $40(63.5)$ \\
\hline Mujer & $23(36.5)$ \\
\hline Edad & \\
\hline $25-30$ & $2(3.2)$ \\
\hline $31-35$ & $4(6.3)$ \\
\hline $36-40$ & $3(4.8)$ \\
\hline $41-45$ & $6(9.5)$ \\
\hline
\end{tabular}




\begin{tabular}{|c|c|}
\hline $\begin{array}{l}46-50 \\
51-55 \\
56-70\end{array}$ & $\begin{array}{l}11(17.5) \\
10(15.9) \\
27(42.9)\end{array}$ \\
\hline $\begin{array}{l}\text { Área de Conocimiento } \\
\text { Estomatología } \\
\text { Oftalmología } \\
\text { Medicina Interna } \\
\text { Anatomía } \\
\text { Dermatología } \\
\text { Fisiología } \\
\text { Ciencias de la Salud } \\
\text { Psiquiatría } \\
\text { Radiología y Medicina Física } \\
\text { Urología } \\
\text { Cirugía General } \\
\text { Traumatología } \\
\text { Otorrrinolaringología } \\
\text { Endocrinología } \\
\text { Anatomía Patológica } \\
\text { Medicina de Familia }\end{array}$ & $\begin{array}{l}20(31.7) \\
3(4.8) \\
1(1.6) \\
2(3.2) \\
1(1.6) \\
4(6.3) \\
18(28.6) \\
2(3.2) \\
2(3.2) \\
3(4.8) \\
2(3.2) \\
1(1.6) \\
1(1.6) \\
1(1.6) \\
1(1.6) \\
1(1.6)\end{array}$ \\
\hline $\begin{array}{l}\text { Experiencia Docente } \\
\qquad \begin{array}{l}\text { 1-5 años } \\
6-10 \text { años } \\
11-15 \text { años } \\
\text { 16-20 años } \\
\text { Más de } 20 \text { años }\end{array}\end{array}$ & $\begin{array}{l}9(14.3) \\
10(15.9) \\
8(12.7) \\
4(6.3) \\
32(50.8)\end{array}$ \\
\hline $\begin{array}{l}\text { Categoría Profesional } \\
\text { Becario PID (proyecto innovación docente) } \\
\text { Catedrático } \\
\text { Colaborador } \\
\text { Interino de Sustitución } \\
\text { PACS } \\
\text { Asociado TP (tiempo parcial) } \\
\text { Ayudante Doctor } \\
\text { Contratado Doctor } \\
\text { Titular }\end{array}$ & $\begin{array}{l}3(4.8) \\
2(3.2) \\
1(1.6) \\
3(4.8) \\
7(11.1) \\
13(20.6) \\
3(4.8) \\
4(6.3) \\
27(42.9)\end{array}$ \\
\hline $\begin{array}{l}\text { Cursos a los que imparte docencia } \\
\text { Sólo } 1 \\
\text { Más de } 1\end{array}$ & $\begin{array}{l}27(42.9) \\
36(57.1)\end{array}$ \\
\hline $\begin{array}{l}\text { Grado-Posgrado } \\
\text { Sólo grado } \\
\text { Grado y Posgrado }\end{array}$ & $\begin{array}{l}44(69.8) \\
19(30.2)\end{array}$ \\
\hline
\end{tabular}




\begin{tabular}{|c|c|}
\hline $\begin{array}{l}\text { ¿Utilizaba antes de la crisis del covid-19 el campus } \\
\text { virtual de manera habitual? } \\
\quad \text { No } \\
\quad \mathrm{Si}\end{array}$ & $\begin{array}{l}31(49.2) \\
32(50.8)\end{array}$ \\
\hline $\begin{array}{l}\text { ¿Cuántos créditos suma el conjunto de estas } \\
\text { materias? } \\
1-6 \\
7-12 \\
13-18 \\
19-24 \\
\text { Más de } 25\end{array}$ & $\begin{array}{l}20(31.7) \\
24(38.1) \\
8(12.7) \\
4(6.3) \\
7(11.1)\end{array}$ \\
\hline 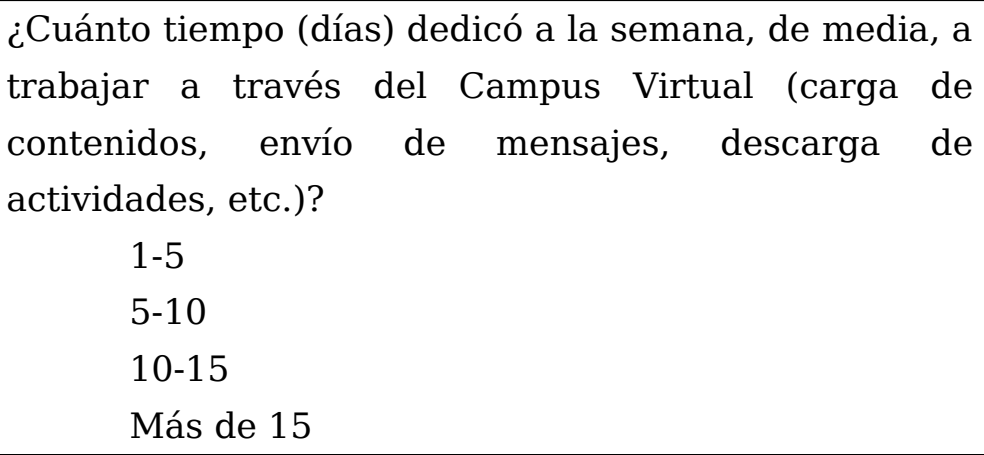 & $\begin{array}{l}40(63.5) \\
14(22.2) \\
5(7.9) \\
4(6.3)\end{array}$ \\
\hline $\begin{array}{l}\text { ¿Con qué frecuencia entró en el Campus Virtual? } \\
\text { Menos de una vez por semana } \\
\text { Una vez por semana } \\
\text { Casi todos los días } \\
\text { Varias veces al día } \\
\text { Varias veces a la semana }\end{array}$ & $\begin{array}{l}19(30.2) \\
12(19) \\
7(11.1) \\
11(17.5) \\
14(22.2)\end{array}$ \\
\hline $\begin{array}{l}\text { ¿Cómo apoya sus asignaturas a través del Campus } \\
\text { Virtual? } \\
\text { No lo uso } \\
\text { Sólo mensajería } \\
\text { Mensajería y material docente } \\
\text { Mensajería, material docente y actividades }\end{array}$ & $\begin{array}{l}4(6.3) \\
2(3.2) \\
33(52.4) \\
24(38.1)\end{array}$ \\
\hline $\begin{array}{l}\text { ¿El Campus Virtual cubre de manera satisfactoria mis } \\
\text { necesidades a las asignaturas que imparto de manera } \\
\text { presencial? (Grado de Acuerdo) } \\
\text { Nada de acuerdo } \\
\text { Algo de acuerdo } \\
\text { De acuerdo } \\
\text { Bastante de acuerdo } \\
\text { Totalmente de acuerdo }\end{array}$ & $\begin{array}{l}9(14.3) \\
5(7.9) \\
15(23.8) \\
26(41.3) \\
8(12.7)\end{array}$ \\
\hline $\begin{array}{l}\text { ¿Qué porcentaje medio de estudiantes matriculados } \\
\text { en las distintas asignaturas entran con regularidad al } \\
\text { Campus Virtual? }\end{array}$ & \\
\hline
\end{tabular}




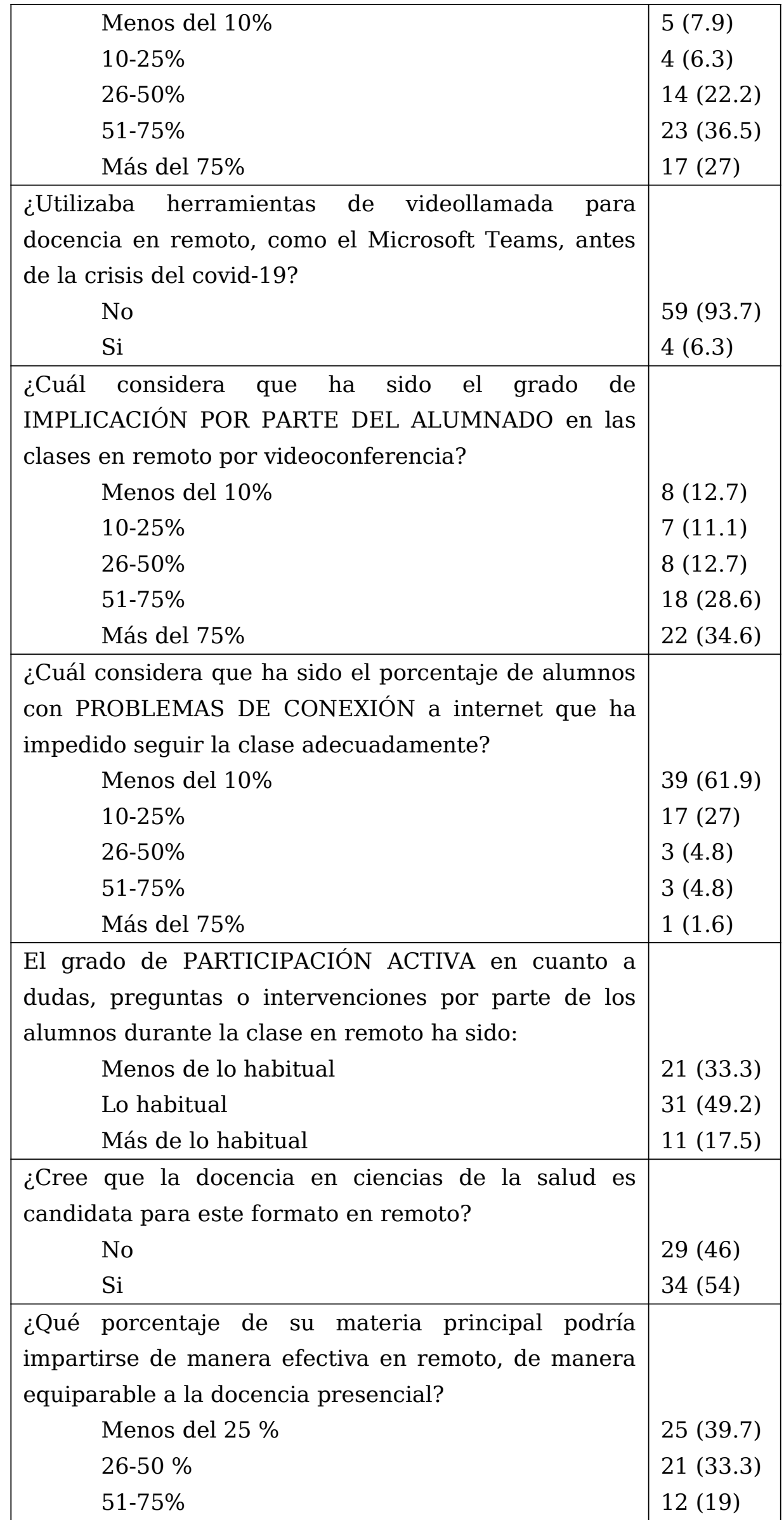




\begin{tabular}{|c|l|}
\hline Más del $75 \%$ & $5(7.9)$ \\
\hline $\begin{array}{l}\text { Señale otros métodos que haya utilizado para la } \\
\text { docencia en remoto o virtual: }\end{array}$ & \\
Ninguno & $2(3.2)$ \\
1 Método & $41(65.1)$ \\
2 Métodos & $9(14.3)$ \\
3 Métodos o más & $11(17.5)$ \\
\hline
\end{tabular}

En relación a su distribución por áreas de conocimiento, el profesorado del área de estomatología suma el 31.7\%, seguido por el $28,6 \%$ de docentes en Ciencias de la Salud (Enfermería, Farmacia, Psicología y Óptica). El 50.8\% cuenta con más de 20 años de experiencia docente. De acuerdo a su categoría profesional, un $3.2 \%$ son profesores catedráticos y un $42.9 \%$ son profesores titulares de universidad (Figura 1). El 57.1\% imparte docencia a más de un curso y casi el $70 \%$ sólo en grado. El 38.1\% asume una carga docente de 7-12 créditos y $11.1 \%$ más de 25 créditos.

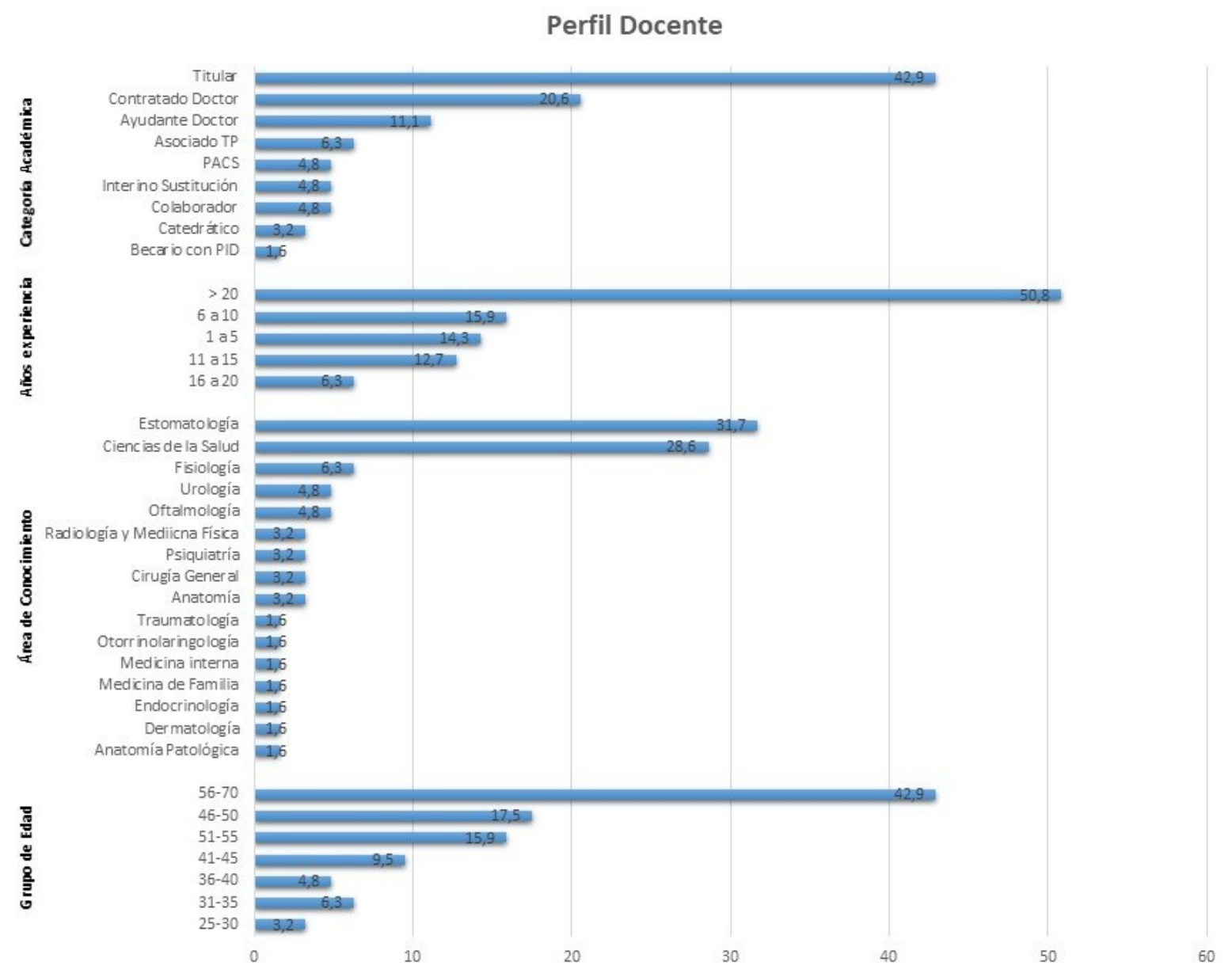

Figura 1. Gráfico con las características del perfil docente de los encuestados: categoría académica, años de experiencia, área de conocimiento y grupo de edad. 
El 49.2\% de los encuestados no utilizaba CV antes y el 6.3\% continúa sin usarlo actualmente. El 63.5\% utilizó CV 1-5 horas semanales, aunque el 6.3\% invirtió más de 15 horas semanales, siendo la frecuencia media mayoritaria (30.2\%) menor a una conexión. En cuanto a la forma de explotación, la mayoría (52.4\%) lo usan como mensajería y material docente y un $38.1 \%$ también para realizar actividades $\mathrm{y}$ autoevaluación. En cuanto a la participación estudiantil el $27 \%$ confirma que participa más del $75 \%$ del alumnado.

En cuanto a la docencia en remoto síncrona, sólo un $6.3 \%$ de los profesores la utilizaba antes. La implicación por parte del alumnado ha sido variable y sólo un 35\% cree que es usado por más del 75\% del alumnado. Casi el 50\% del profesorado cree que la participación es la habitual comparado con la docencia presencial, pero el $17.5 \%$ ha percibido un incremento de participación. Se han registrado problemas de conectividad y el $62 \%$ del profesorado considera una afectación de menos del $10 \%$ del alumnado incluso un $1.6 \%$ considera más del $75 \%$. Se empleó mayoritariamente Microsoft Teams recomendada institucionalmente, aunque el $14.3 \%$ usó dos métodos y el $17.5 \%$ tres o más (Figura 2).

En relación a la satisfacción con CV y la cobertura de necesidades en las materias impartidas, el $14.3 \%$ del profesorado no está nada de acuerdo. El $54 \%$ de los encuestados considera la docencia en remoto válida para su aplicación en Ciencias de la Salud, pero un 39.7\% sólo podría aplicarlo a menos del $25 \%$ de la/s materia/s como método único. Constatamos de manera estadísticamente significativa, que los profesores asociados TP son los que menos usaban CV antes de la crisis sanitaria, un 29\% de todos los no usuarios; mientras los profesores titulares suponen un 56.3\% de todos los usuarios habituales $\left(\mathrm{chi}^{2}=15.816, \mathrm{~V}\right.$ de Cramer $\left.=0.501, \mathrm{p}=0.045\right)$. Los profesores usuarios CV antes de la crisis, son los que presentaron mayor frecuencia semanal $\left(\mathrm{chi}^{2}=13.774, \mathrm{~V}\right.$ de Cramer $\left.=0.468, \mathrm{p}=0.008\right)$. Los no usuarios, mayoritariamente (64.5\%) comienzan a usarlo como mensajería y entrega de material docente, mientras entre los usuarios habituales un 59.4\% explotan todas sus posibilidades ( $\operatorname{chi} 2=15.640, \mathrm{~V}$ de Cramer $=0.498, \mathrm{p}=0.001$ ). Los usuarios CV imparten de manera estadísticamente significativa más materias, $2.5 \pm 2.2$ vs $1.4 \pm 1.5$ materias los no usuarios $(\mathrm{p}=0.017)$. En cuanto a la docencia en remoto, sólo era utilizada por el $6.3 \%$ del profesorado, de éstos el $100 \%$ la consideraron adecuada para Ciencias de la Salud, aunque con dependencia moderada, frente al $49.2 \%$ que no la consideraron apta $\left(\mathrm{chi}^{2}=3.643, \mathrm{~V}\right.$ de Cramer $\left.=0.240, \mathrm{p}=0.05\right)$. En esta misma línea, consideraron adecuado este formato el $65 \%$ de los hombres vs $34.8 \%$ de las mujeres (chi2=5.367, $\mathrm{p}=0.021$; $\mathrm{V}$ de Cramer=0.292, $\mathrm{p}=0.021$ ). 


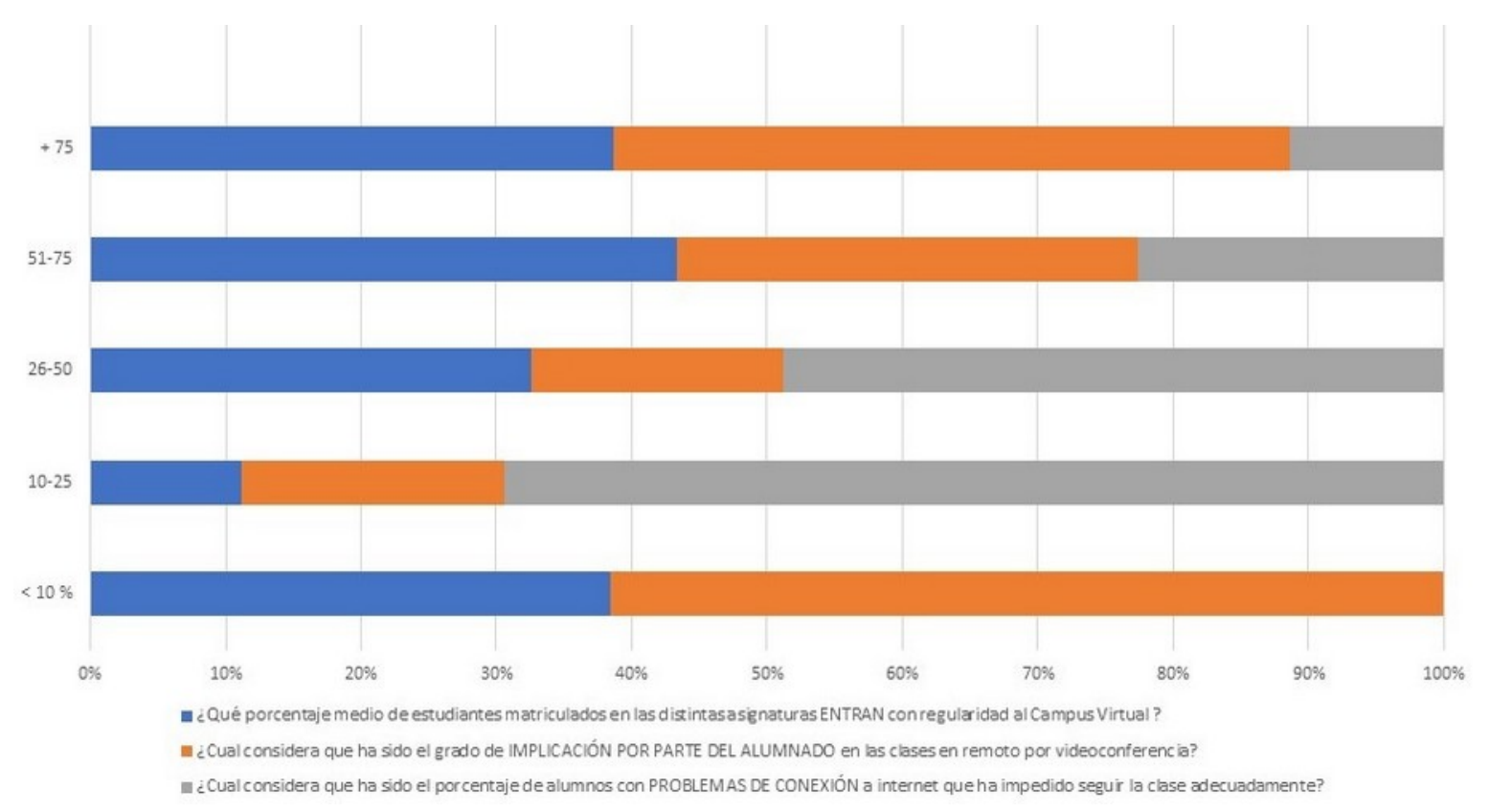

Figura 2. Representación gráfica sobre aspectos del uso del CV

\section{Discusión}

El aprendizaje electrónico (Internet para mejorar el conocimiento y el rendimiento) es un enfoque educativo ampliamente aceptado. La pandemia Covid-19 ha impulsado cambios más rápidos en la educación superior exigidos por las medidas de distanciamiento social que impiden cualquier enseñanza presencial. Según Manzanares et al. (17) el entorno virtual (uso de infografías y laboratorios virtuales), es en el que los estudiantes de ciencias de la salud obtienen mayor grado de satisfacción. Sin embargo, desconocemos la percepción por el profesorado hacia estas metodologías, por lo que vamos a desarrollar los aspectos más relevantes que pueden determinar el uso del VLE: aspectos sociales, económicos situacionales, tecnológicos y aquellos inherentes a las propias áreas de conocimiento.

Nekenke et al. (18), afirmaron que la aceptación de las nuevas tecnologías está influenciada por una serie de factores situacionales y sociales. En nuestra muestra, los hombres mayoritariamente consideran el VLE sincrónico adecuado para la docencia en Ciencias de la Salud. Este hecho puede estar relacionado con la baja participación de las mujeres en la encuesta y que está condicionada a su vez por el número de profesoras en los centros universitarios encuestados. Si bien es cierto que se trata de un tamaño muestral limitado para poder hacer inferencias generales. Nuestro estudio constata un bajo uso del CV y de la docencia en remoto, ligado a la elevada edad media del profesorado, al igual que Marina et al. (19), donde la edad parece ser un factor clave en el uso de las nuevas tecnologías.

Un 50.8\% de los encuestados usaba CV de manera habitual y asíncrona. Wittich et al. (15) a través de una encuesta en USA a coordinadores de programas de posgrado en Medicina Interna, el 71,5\% utilizaban el aprendizaje electrónico asíncrono con cierta frecuencia, siendo el aspecto 
económico determinante para su implementación. Dombrowski et al. (20) sobre la enseñanza digital observó que ésta se ve obstaculizada por la falta de financiación y menos por las condiciones técnicas. Este factor económico parece ser también decisivo en el entorno en que se ha desarrollado este trabajo.

Nuestros resultados constatan problemas de conectividad en el $75 \%$ del alumnado, lo que supone una limitación para la enseñanza y la evaluación en VLE. Al-Neklawy et al. (21), en la enseñanza de embriología general, encontraron altas dificultades de conexión a la plataforma. Hart et al. (22) encontraron similares problemas técnicos en enfermería. La participación del alumnado en VLE sincrónico a juicio de los encuestados es el habitual (49.2\%) o incluso superior (17.5\%), aunque varía dependiendo de las áreas de conocimiento y el uso previo por parte del profesorado. No todas las áreas de conocimiento presentan las mismas necesidades, Longhurst et al. (24) afirmaron que en anatomía existen desafíos singulares debido a la tridimensionalidad. Comer et al. (23) consideraron preciso adaptar el programa práctico a VLE. Igualmente, en estudiantes de odontología, la capacitación basada en pacientes virtuales y otras herramientas digitales, puede mejorar el aprendizaje y la capacidad de toma de decisiones (24-27) sobre todo en el ámbito pre-clínico (28). Así Mardani et al. (29), en un estudio reciente sobre capacitación virtual (intervención) y presencial (control), la puntuación media en la toma de decisiones clínicas en el grupo virtual fue significativamente mayor que el control. Por otra parte, un programa de aprendizaje para docentes e intervención tempranas parecen ser la clave para evitar problemas derivados del uso abrupto y no coordinado de VLE.

Este trabajo presenta una serie de limitaciones inherentes a la propia metodología, fundamentalmente relacionadas con las encuestas autoaplicadas y la baja participación. Tratándose de un estudio piloto en nuestra Universidad, sería necesario realizar mecanismos de validación o control interno de coherencia de la encuesta para poder determinar el grado de acuerdo. Sin embargo, creemos que la baja participación, puede ser reflejo de la grave situación de presión que todas las unidades docentes y su profesorado ha sufrido y actualmente siguen padeciendo, por la abrupta implantación de la docencia no presencial.

\section{Conclusiones}

- El distanciamiento social condiciona forzosamente el uso de VLE en Ciencias de la Salud y en algunas áreas de conocimiento, su uso no había sido protocolizado antes de la crisis del covid-19.

- La utilización de los recursos virtuales es baja, siendo la docencia sincrónica la menos utilizada.

- En cuanto a las prestaciones ofrecidas por el campus virtual, parecen poco explotadas por los docentes encuestados incluso durante el periodo de docencia no presencial.

- La baja utilización del campus y de la docencia virtual obtenida, sugiere una necesidad de capacitación en VLE por el profesorado en ciencias de la salud, mejorando e incrementando su uso.

Financiación: No ha habido financiación. 
Declaración de conflicto of interés: Los autores declaran no tener ningún conflicto de intereses.

\section{Referencias}

1. Pather N, Blyth P, Chapman JA, Dayal MR, Flack, Natasha A. M. S., Fogg QA, Green RA, Hulme AK, Johnson IP, Meyer AJ, Morley JW, Shortland PJ, Štrkalj G, Štrkalj M, Valter K, Webb AL, Woodley SJ, Lazarus MD. Forced Disruption of Anatomy Education in Australia and New Zealand: An Acute Response to the Covid-19 Pandemic. Anat Sci Educ. 2020; 13(3): 284-300. http://doi.org/10.1002/ase.1968

2. Blackmon B. Social work and online education with all deliberate speed. J Evid Based Soc Work. 2013;10(5):509-21. http://doi.or/10.1080/15433714.2012.663672.

3. Riley JB, Austin JW, Holt DW, Searles BE, Darling EM. Internet-based virtual classroom and educational management software enhance students' didactic and clinical experiences in perfusion education programs. J Extra Corpor Technol. 2004;36(3):235-9. https://pubmed.ncbi.nlm.nih.gov/15559740/

4. Blondel DV, Sansone A, Rosenberg J, Godin EA, Yang BW, Jaglom-Kurtz LT, LinnenbrinkGarcia L, Schwartz-Bloom RD. Development of an Online Experiment Platform for High School Biology. J Form Des Learn. 2019;3(1):62-81. http://doi.org/10.1007/s41686-01900030-5

5. Bigony L. Can you go the distance? Attending the virtual classroom. Orthop Nurs. 2010; 29(6):390-392. http://doi.org/10.1097/NOR.0b013e3181f8380a

6. Swartzwelder K, Clements P, Holt K, Childs G. Confronting Incivility in the Online $\begin{array}{llll}\text { Classroom. } & \text { J }\end{array}$ http://doi.org/10.1097/CNJ.0000000000000591

7. Madrigal E, Mannan R. pathCast: An Interactive Medical Education Curriculum That Leverages Livestreaming on Facebook and YouTube. Acad Med. 2020;95(5):744-750. http://doi.org/10.1097/ACM.0000000000003148

8. Sharma N, Doherty I, Dong C. Adaptive Learning in Medical Education: The Final Piece of Technology Enhanced Learning? Ulster Med J. 2017;86(3):198-200. https://pubmed.ncbi.nlm.nih.gov/29581634/

9. Handal B, Groenlund C, Gerzina T. Academic perceptions amongst educators towards eLearning tools in dental education. Int Dent J. 2011;61(2):70-75. http://doi.org/10.1111/j.1875-595X.2011.00017.x

10. Cordero Torres JA, Caballero Oliver A. [The Moodle platform: A useful tool for training in life support. Analysis of satisfaction questionnaires from students and instructors of the semFYC advanced life support courses]. Aten Primaria. 2015;47(6):376-84. http://doi.org/ 10.1016/j.aprim.2015.02.006

11. Popovic N, Popovic T, Rovcanin Dragovic I, Cmiljanic O. A Moodle-based blended learning solution for physiology education in Montenegro: a case study. Adv Physiol Educ. 2018;42(1):111-117. http://doi.org/10.1152/advan.00155.2017

12. Memon AR, Rathore FA. Moodle and Online Learning in Pakistani Medical Universities: An opportunity worth exploring in higher education and research. J Pak Med Assoc. 2018;68(7):1076-1078. https://pubmed.ncbi.nlm.nih.gov/30317305/

13. Carney PA, Mejicano GC, Bumsted T, Quirk M. Assessing learning in the adaptive $\begin{array}{llllll}\text { curriculum. } & \text { Med } & \text { Teach. } & \text { 2018; } & 40 & \text { (8): }\end{array}$ http://doi.org/10.1080/0142159X.2018.1484083

14. Chu LF, Young CA, Ngai LK, Cun T, Pearl RG, Macario A. Learning management systems and lecture capture in the medical academic environment. Int Anesthesiol Clin. 2010;48(3):27-51. http://doi.org/10.1097/AIA.0b013e3181e5c1d5

15. Wittich CM, Agrawal A, Cook DA, Halvorsen AJ, Mandrekar JN, Chaudhry S, Dupras DM, Oxentenko AS, Beckman TJ. E-learning in graduate medical education: survey of residency program directors. BMC Med Educ. 2017;17(1):114. http://doi.org/10.1186/s12909-017$\underline{0953-9}$ 
16. Manchikanti L, Singh V, Smith HS, Hirsch JA. Evidence-based medicine, systematic reviews, and guidelines in interventional pain management: part 4: observational studies. Pain Physician. 2009;12(1):73-108. https://pubmed.ncbi.nlm.nih.gov/19165298/

17. Sáiz Manzanares MC, García-Osorio CI, Díez-Pastor JF. Differential efficacy of the resources used in B-learning environments. Psicothema. 2019;31(2):170-178. http://doi.org/10.7334/psicothema2018.330

18. Nkenke E, Vairaktaris E, Schaller H, Perisanidis C, Eitner S. Influence of gender of the teaching staff on students' acceptance of a virtual implant planning course. J Craniomaxillofac Surg. 2017;45(5):614-619. http://doi.org/10.1016/j.jcms.2017.01.026

19. Marina Ocaña J, Feliz Murias T. [Perceptions about the Information and Education for Health in Virtual Environments in Spanish]. Rev Esp Salud Publica. 2018;92:e201808022. https://pubmed.ncbi.nlm.nih.gov/30066699/

20. Dombrowski T, Dazert S, Volkenstein S. Strategies of Digitized Learning. Laryngorhinootologie. 2019;98(S 01):S197-S219. http://doi.org/10.1055/a-0803-0218

21. Al-Neklawy AF. Online Embryology teaching using learning management systems appears to be a successful additional learning tool among Egyptian medical students. Ann Anat. 2017; 214:9-14. http://doi.org/10.1016/j.aanat.2017.07.001

22. Hart T, Bird D, Farmer R. Using blackboard collaborate, a digital web conference tool, to support nursing students placement learning: A pilot study exploring its impact. Nurse Educ Pract. 2019; 38: 72-78. http://doi.org/10.1016/j.nepr.2019.05.009

23. Comer BT, Gupta N, Mowry SE, Malekzadeh S. Otolaryngology Education in the Setting of COVID-19: Current and Future Implications. Otolaryngol Head Neck Surg. 2020;163(1):70-

74. http://doi.org/10.1177/0194599820923621

24. Thilakumara IP, Jayasinghe RM, Rasnayaka SK, Jayasinghe VP, Abeysundara S. Effectiveness of Procedural Video Versus Live Demonstrations in Teaching Laboratory Techniques to Dental Students. J Dent Educ. 2018;82(8):898-904. http://doi.org/10.21815/JDE.018.086

25. Abdul-Razzak S. Evaluation of the first year of Dental Health Partnerships: a web-based distance learning partnership between UK dental educators and students from lowresource countries. Br Dent J. 2018;225(3):252-256. http://doi.org/10.1038/sj.bdj.2018.545

26. Mattheos N, Nattestad A, Schittek M, Attström R. A virtual classroom for undergraduate periodontology: a pilot study. Eur J Dent Educ. 2001;5(4):139-47. http://doi.org/10.1034/j.1600-0579.2001.50401.x

27. Chen Y, Hsue S, Lin D, Wang W, Chen J, Lin C, Lin L. An application of virtual microscopy in the teaching of an oral and maxillofacial pathology laboratory course. Oral Surg Oral Med Oral Pathol Oral Radiol Endod. 2008;105(3):342-7. http://doi.org/10.1016/j.tripleo.2007.03.020

28. Towers A, Field J, Stokes C, Maddock S, Martin N. A scoping review of the use and application of virtual reality in pre-clinical dental education. Br Dent J. 2019;226(5):358366. http://doi.org/10.1038/s41415-019-0041-0

29. Mardani M, Cheraghian S, Naeeni SK, Zarifsanaiey N. Effectiveness of virtual patients in teaching clinical decision-making skills to dental students. J Dent Educ. 2020;84(5):615623. http://doi.org/10.1002/jdd.12045

(C) 2020 por los autores. Enviado para su publicación en acceso abierto bajo los términos y condiciones de la licencia Creative Commons Attribution (CC BY) (http://creativecommons.org/licenses/by/4.0/). 